\title{
Review of: "Multiple knockout mutants reveal a high redundancy of phytotoxic compounds that determine necrotrophic pathogenesis of Botrytis cinerea"
}

\author{
LUCA SELLA $^{1}$, Arianna Panichi ${ }^{1}$ \\ 1 University of Padua
}

Potential competing interests: The author(s) declared that no potential competing interests exist.

The paper deals with the creation and the characterization of multiple mutants of $B$. cinerea for genes encoding plant cell death inducing proteins (CDIPs) involved in the necrotic process of plant tissues induced by $B$. cinerea infection. In particular, through an innovative mutagenesis technique based on the CRISPR/CAS9 system the authors managed to obtain a mutant of $B$. cinerea with up to 12 different CDIP encoding genes disrupted. The growth of the mutants was comparable to WT, but infection experiments showed a reduction of the fungal virulence as the number of mutations increased. This was particularly true when the genes for endopolygalacturonases (pg1 and pg2) and for two major phytotoxic metabolites, botrydial and botcnin, were mutated. The study contains a huge amount of data that clearly demonstrate the redundancy of virulence factor (protein and metabolites) in the fungal pathogen $B$. cinerea.

\section{General comment:}

Overall, the manuscript is very interesting since it characterizes for the first time the functional redundancy of virulence factors of a fungal pathogen using an innovative molecular gene knock-out approach and surely deserves to be published.

Except for some misspellings or some unclear sentences, the paper is well written. However, I found a critical point that should be clarified by authors: in particular, it is not very clear how multiple mutants were obtained. In fact, what is reported in the section "Materials and Methods- DNA manipulations" differs from what is reported in Table 2 about the order followed to obtain the serial deletion mutants. Other specific minor comments are reported here below.

\section{Minor comments:}

Lines 17-18: The sentence is not well written and should be rewritten

Lines 72-73: it is not clear what IEB and Hip1 are. Please include a short description as done for other CDIPS

Line 85: in fact, Ron and Avni (2004) report the identification of the receptor of EIX, a Trichoderma xylanase, and not that of BcXyn11A. 
Line 140: the reference for xyl1 mutant is (Yang et al. 2018), not (Noda et al. 2010)

Line 142: authors write "as shown below" but there is no reference to a figure or a table.

Lines 182-187: it might be interesting for the reader to understand why the authors decided to make two different $12 x$ mutants.

Line 190: from Fig. 2A the mutant $4 x^{r}$ seems significantly different from WT.

Line 256: Please indicate which proteins were not detected in the WT secretome to facilitate the reader. Lines 264-266: Were the on planta secretome data uploaded in a public database?

Lines 351-352: I suggest to reformulate as follows: "lesion size > 60\% compared to WT".

Lines 415-419: I suggest to reformulate the sentences: "For growth tests, agar plates containing Gamborg minimal medium (GB5) containing $25 \mathrm{mM}$ glucose. For sporulation tests, $10 \mu \mathrm{l}$ droplets containing $10^{5}$ conidia $\mathrm{ml}^{-1}$ were inoculated onto ME agar plates, and incubated at $20-22^{\circ} \mathrm{C} . "$

Lines 467-470: This description of the quadruple mutant is in contrast from what reported in Table 2.

Line 481: Malus domestica should be in italics

Line 486: How many conidia were used for apple fruit inoculation?

Line 498: which Nicotiana benthamiana cultivar did the authors use?

Lines 503-504: how did the authors concentrate the fraction?

Line 510: it is not clear why the authors killed the onion epidermal layers for microscopic analysis. Please provide an explanation.

Line 512: droplets “containing" $5 * 10^{4}$ conidia $\mathrm{ml}^{-1}$

Line 516: Did the authors performed washing with water?

Line 527: the reference to Figure 2 is not correct.

Figure 1A: the description in the legend is incomplete. Please provide more details.

Figure 2E: The description of the acronyms used in the images are missing.

Figure 3: I would suggest to replace h with "hpi" in the legend.

Figure S2: I would reformulate the first line of the description as follows: "PCR-based confirmation of gene deletions in the 12 xbb mutant and mapping of pg1 and pg2 deletions on the $12 x p g$ mutant". Why did authors perform the PCR analysis using as template the 12xbb mutant and not the 10x mutant, which was then used as background for obtaining both 12xbb and 12xpg? Besides, the sketch should be explained better, since it allows to understand all the PCR results reported. Finally, authors refer to Table S4 (line 739), which should be Table S3 instead.

Table S1: This table is difficult to understand. What do "size (change)" and "deleted codons" columns mean? I recommend authors to describe it better.

Table S2: MS/MS-detection is quite strange in the case of PG1 and PG2. As expected, their level is very low on the pg1pg2 and 12xpg mutants, compared to WT. But what about the 11x mutant? Why also the PG2 level is so low when only the pgl gene was deleted? Authors should try to give an explanation for this strange observation. 
